\title{
Association between offspring birth weight and atherosclerosis in middle aged men and women: British Regional Heart Study
}

\author{
D A Lawlor, G Davey Smith, P Whincup, G Wannamethee, O Papacosta, S Dhanjil, \\ M Griffin, A N Nicolaides, S Ebrahim
}

in ow birth weight is associated with cardiovascular disease in adulthood, but the mechanisms underlying these associations remain unclear. ${ }^{1}$ One possibility is that poor intrauterine nutrition "programmes" adult disease. ${ }^{1}$ An alternative hypothesis is that specific polymorphisms with pleiotrophic effects result in both small birth size and cardiovascular disease risk. ${ }^{2}$ Epidemiological evidence for a genetic role in the association comes from the recent finding that offspring birth weight predicts parental cardiovascular disease risk. ${ }^{34}$ As an infant's low birth weight cannot determine its own parents' risk of cardiovascular disease through intrauterine programming, a plausible explanation for these trans-generational associations is that of a common genetic link. In this paper we investigate the associations between offspring birth weight and parental carotid intima media thickness, an indicator of pre-clinical disease.

\section{METHODS AND RESULTS}

Data were used from the Dewsbury and Maidstone cohort of the British Regional Heart Study. B-mode ultrasound measurements of carotid arteries, together with a detailed physical examination, blood tests, and research nurse interview were undertaken on 375 women and 425 men, aged 56-75 years. Full details of all measurements have been reported previously. In the absence of atherosclerotic plaque carotid intima media thickness was measured at the bulb origin, which was defined as the point at which the arterial wall diverges to form the bulb. In the presence of plaque, the maximum thickness of the plaque was measured and this was taken as the intima media thickness. This measure provides an indication of pre-clinical atherosclerosis. ${ }^{5}$ For participants with children details of the birth weight and sex of their first offspring were requested. Multiple linear regression was used to assess the association between offspring birth weight and intima media thickness, with adjustment for potential confounding factors. Intima media thickness measurements had a skewed distribution but logged values were normally distributed; logged values were used in the linear regression models.
Altogether 375 women and 425 men attended (69\% and $83 \%$ response respectively); of these adequate ultrasound images were obtained on 367 women and 418 men. A total of 268 women and 221 men had at least one offspring; all provided data on their birth weight.

Table 1 shows the association between offspring birth weight and intima media thickness with adjustment for potential confounding factors. Intima media thickness was inversely associated with offspring birth weight in both women and men, though the estimates were imprecise with wide confidence intervals. Adjustment for body mass index and further adjustment for social class and smoking did not affect the magnitude of these associations.

\section{DISCUSSION}

We have shown that offspring birth weight is inversely associated with carotid artery intima media thickness. Though small numbers make the results of our study imprecise these results are consistent with other studies. ${ }^{34}$ The association between offspring birth weight and carotid intima media thickness in both women and men cannot be explained by confounding attributable to maternal menstrual or reproductive factors, and is consistent with the association between offspring birth weight and coronary heart disease that has been seen in both sexes. ${ }^{3}$ Birth weight is largely determined by environmental factors and a plausible explanation for the association between birth weight and maternal intima-media thickness would be that maternal environmental factors such as poor diet affect both her own risk of atherosclerosis and her offspring's birth weight. However, the association between offspring birth weight and paternal atherosclerosis is not so readily explained. A plausible explanation for these transgenerational associations is a common genetic factor associated with both lower birth weight and higher risk of atherosclerosis in later life.

We relied on self report of offspring birth weight. Most of the offspring in this study were born in the mid to late 1950s and the mean birth weights in this study were similar to those for the 1958 British birth cohort: $3.40 \mathrm{~kg}$ ( SD $0.45 \mathrm{~kg}$ ) for boys

Table 1 Association between offspring birth weight and carotid IMT

\begin{tabular}{lllll}
\hline & $\begin{array}{l}\text { Number with complete } \\
\text { data on all variables } \\
\text { included in models }\end{array}$ & $\begin{array}{l}\text { Age adjusted regression } \\
\text { coefficient logged IMT/g } \\
\text { offspring birth weight }\end{array}$ & $\begin{array}{l}\text { Age and BMI adjusted } \\
\text { regression coefficient logged } \\
\text { IMT/kg offspring birth weight }\end{array}$ & $\begin{array}{l}\text { Age, BMI, social class and smoking } \\
\text { adjusted regression coefficient } \\
\text { logged IMT/kg offspring birth weight }\end{array}$ \\
\hline All & 483 & $-0.04(-0.11$ to 0.02$)$ & $-0.04(-0.11$ to 0.02$)$ & $-0.04(-0.11$ to 0.03$)$ \\
Women & 267 & $-0.04(-0.14$ to 0.05$)$ & $-0.04(-0.14$ to 0.05$)$ & $-0.04(-0.14$ to 0.05$)$ \\
Men & 202 & $-0.03(-0.13$ to 0.06$)$ & $-0.03(-0.12$ to 0.07$)$ & $-0.03(-0.13$ to 0.07$)$ \\
\hline
\end{tabular}

BMI, body mass index $\left(\mathrm{kg} / \mathrm{m}^{2}\right)$; smoking categorised as never, ex, current; social class derived from longest held social class and classified according to registrar generals classification - I, II, III non-manual, III manual, IV, V. In all models robust standard errors were used to calculate $95 \%$ confidence intervals taking into account the clustering effect of each town. 
in the 1958 cohort compared with $3.35 \mathrm{~kg}$ (SD $0.51 \mathrm{~kg}$ ) for boys in our study, and $3.26 \mathrm{~kg}$ ( SD $0.43 \mathrm{~kg}$ ) for girls in the 1958 cohort compared with $3.26 \mathrm{~kg}$ (SD $0.54 \mathrm{~kg}$ ). Our data seem to be representative of the general population, and reported offspring birth weight data seem valid.

Our findings and those of other studies ${ }^{3}$ provide epidemiological support for the hypothesis that common genetic factors are important in the association between birth weight and cardiovascular disease. Future research should attempt to identify specific candidate genes.

\section{ACKNOWLEDGEMENTS}

We are grateful to the two general practices who collaborated in the field work, to our field team (Stella Barlow RGN, Annalise Hamilton RGN, Lucy Lennon BSc) who carried out the field work and all the participants of the British Regional Heart Study.

\section{Contributors}

DAL, GDS, and SE developed the idea for this study and SE, PW, GW, and OP set up and organised data collection for the Maidstone Dewsbury cohort. SD and MG undertook the arterial ultrasound scans and ANN interpreted the arterial ultrasound videos. DAL undertook the statistical analysis. DAL wrote the first draft of the paper and all authors have contributed to the final version. All authors act as guarantors.

\section{Authors' affiliations}

D A Lawlor, G Davey Smith, S Ebrahim, Department of Social Medicine, University of Bristol, Bristol, UK

P Whincup, Department of Public Health Sciences, St George's Hospital Medical School, London, UK

G Wannamethee, O Papacosta, Department of Primary Care and
Population Sciences, Royal Free Hospital School of Medicine, London, UK

S Dhaniil, M Griffin, A N Nicolaides, Division of Surgery, Anaesthetics and Intensive Care, Irvine Laboratory, Imperial College School of Medicine, St Mary's Hospital, London, UK

A N Nicolaides, Department of Neurovascular Sciences, The Cyprus Institute of Neurology and Genetics, Nicosia, Cyprus

Funding: This study was funded by the Stroke Association. The Department of Health and British Heart Foundation have provided funding for the British Regional Heart Study. The CDER Trust provided funding for the ultrasound equipment used in this study. DAL is funded by the Medical Research Council. The opinions expressed are those of the authors.

\section{Conflicts of interest: none.}

Correspondence to: Dr D A Lawlor, Department of Social Medicine, University of Bristol, Canynge Hall, Whiteladies Road, Bristol BS8 2PR, UK; d.a.lawlor@bristol.ac.uk

Accepted for publication 24 August 2002

\section{REFERENCES}

1 Barker DJP. Mothers, babies and health in later life. London: Churchill Livingstone, 1998.

2 Hattersley AT, Tooke JE. The fetal insulin hypothesis: an alternative explanation of the association of low birthweight with diabetes and vascular disease. Lancet 1999;353:1789-92.

3 Davey Smith G, Hart C, Ferrell C, et al. Birth weight of offspring and mortality in the Renfrew and Paisley study: prospective observational study. BM 1997;315:1189-93.

4 Davey Smith G, Harding S, Rosato M. Relation between infants' birth weight and mothers' mortality: prospective observational study. BM 2000;320:839-40.

5 Ebrahim S, Papacosta O, Whincup P, et al. Carotid plaque, intima media thickness, cardiovascular risk factors, and prevalent cardiovascular disease in men and women: the British Regional Heart Study. Stroke 1999;30:841-50. 